overlap with the companion volume Satellites of Jupiter.

The first eight chapters give a complete and uniformly excellent review of the observations. These chapters alone are worth the price of the book. The remaining four chapters are theoretical and vary widely in scope, depth, and detail. A unique bonus is the appendix on Jovian coordinate systems, which gives a useful explanation of the many Jovian latitude and longitude conventions. The level of the book, particularly in the observational chapters, is suitable for graduate students and research workers outside the field. Some of the material in the theoretical chapters will be fully appreciated only by experts in plasma theory.

It might have been helpful to provide an introductory overview chapter, which would have given a survey of the main phenomenology, assessed the current status of the field, indicated some directions for future development, and finally discussed implications for other fields in astrophysics and space physics. The individual chapters in the book do provide summary and discussion sections, which discuss some of these topics, but within the context of the separate subfields.

In short, this book is a unique and invaluable resource, which should be considered an essential acquisition for libraries and individuals interested in space plasmas and plasma astrophysics. It is also highly recommended for astrophysicists and space physicists in general.

Andrew F. Cheng is with the Applied Physics Laboratory, The Johns Hopkins University, Laurel, MD 20707

\section{Satellites of Jupiter}

D. Morrison (Ed.), University of Arizona Press, Tucson, Arizona, $x+972$ pp., 1982, $\$ 49.50$

\section{PAGES 507-508}

\section{Reviewed by D.J. Stevenson}

Future historians of science will look back on the arrivals of the Voyager spacecraft at the Jupiter system in 1979 as very significant events, primarily because of the remarkable diversity of new phenomena discovered on the Galilean statellites. In a short time period there was almost a doubling of the number of solid "planetary" bodies for which substantial scientific analysis is possible. Scientists were introduced to the novelties of tidally heated bodies and of ice tectonics. From a more fundamental point of view, there is much to be learned about the origin and evolution of planets and their environments from analysis of the satellite systems. Any doubts about the importance of studying planetary satellites must have been dispelled by the Voyager missions.

A meeting was held in Hawaii, May 1980 , primarily to present and to discuss the results of the Voyager observations of the Jovian satellites. Arising from the meeting came this new book, another in the excellent Space Science Series of volumes published by the Unviersity of Arizona Press. Edited by David Morrison with the assistance of Mildred Shapley Matthews, Satellites of Jupiter involves 47 collaborating authors, 24 chapters, and is for the most part impressively comprehensive and authoritative. Perhaps inevitably it is also probably the least successful in this series of volumes. Even this is a mild criticism because of the high standards achieved and maintained by these books.

The problem lies in the nature of the subject matter and the circumstances in which this book was produced. Whereas most previous volumes (e.g., Planetary Satellites, Asteroids) consisted primarily of thoughtful, broad, and integrated reviews of well-established subject matter, authors in Satellites of Jupiter were confronted with the task of digesting an enormous data set and presenting overviews of new science in a short period of time. Most of the chapters were finished less than 18 months after the Voyager encounters; a late chapter or two greatly delayed publication until 1982. As a consequence of the limited time and the novelty of the science, some of the chapters read more like journal papers than review articles. This reviewer also suspects that many of the theoretical interpetations presented will be (or are being) superseded. In fact, most of the fundamental questions posed by the Voyager results remain unanswered. For example, the heat flow of Io is not quantitatively understood (although the tidal heating proposed by Peale et al. is not seriously in doubt), the nature of Europa's surface and outer regions remains enigmatic, no entirely satisfactory explanation yet exists for the remarkable surficial dissimilarity of Ganymede and Callisto, all aspects of the satellite histories (orbital evolution, cratering, internal structure, surface modification, atmospheric) remain controversial, the geochemistry of Io's volcanism is puzzling, and the dynamics of the Io plasma torus remain unclear. Controversy and uncertainty are the lifebood of science, but the level of ignorance may not be apparent to the reader confronted with 972 pages of information overload.

This book is nevertheless indispensible to the planetary scientist and invaluable to the graduate student or researcher entering (or contemplating) this area. Around $40 \%$ of the text deals with Io, an appropriate fraction because of the diversity of phenomena related to it. The other Galilean satellites also receive extensive coverage, and separate chapters are devoted to the rings of Saturn, Amalthea, the outer satellites, and the Io torus. However, most of the chapters deal with physical phenomena rather than with specific bodies.

Notable chapters include S. W. Kieffer's very thorough (although possibly too detailed) effort on the dynamics and thermodynamics of volcanic eruptions; no comparable effort exists anywhere else. The effort by Shoemaker and Wolfe, on cratering time scales, is also a remarkable if somewhat controversial synthesis of existing data and theory. Ostro's chapter on the radar observations of the icy satellites is interesting becuase it offers a tantalizing glimpse of the nature of the uppermost few meters, potentially very important for understanding the compositional and tectonic evolutions of these bodies. The chapter on atmospheres by Kumar and Hunten is succinct yet thorough, and the geological chapters (Ganymede by Shoemaker et al., Europa by Lucchitta and Soderblom, Io by Shaber) are uniformly well written and good at the descriptive level (but occasionally faltering at the interpretive level).

Typical of the University of Arizona Sijuce
Science Series, this text is well produced, with a small (but finite) density of typographical errors and a substantial but understandable price in view of the bulk. It could have benefited from stronger editorial control to reduce length, and it also suffers from a small but significant number of poorly reproduced Voyager images or maps (even allowing for the limitations of non-glossy paper). It will remain a very useful text for many years, if only because of the infrequency of deep space missions.

D. J. Stevenson is with the Division of Geological and Planetary Sciences, California Institute of Technology, Pasadena, CA 91125.

\section{Physique Moleculaire: Physique de l'Atmosphere}

C. Camy-Peyret (Ed.), Editions du Centre National de la Recherche Scientifique, Paris, 502 pp., 1982.

\section{PAGE 508}

\section{Reviewed by Marcel Ackerman}

Physique Moleculaire: Physique de l'Atmosphere is a collection of lectures presented at a winter school on the "Application of Molecular Physics to the Atmosphere and to the Environment," organized from December 1-10, 1983 , in Montfoulon (Normandy) under the auspices of various French governmental agencies including the National Center for Scientific Research (CNRS), the Center for Nuclear Studies, and the National Center for Space Studies (CNES). This initiative is part of a policy which developed a few years ago in France and is intended to promote interdisciplinary activities in order to foster interdisciplinary research.

Since the early 1970 s, several serious questions have been put to the scientific community concerning the possible effects of man's activities on the atmosphere and the likely impact these effects have on the climate. The goal of these lectures was to encourage French scientific communities active in aeronomy, chemical kinetics, meterology, and spectroscopy to work together on those questions of upper atmosphere-climate relationships, since they offer a typical interdisciplinary character. Most of the 12 lectures are in French, except for two that are in English, and can be devided into two groups: introductions of the structure of the atmosphere, the photochemistry and spectroscopy of atmospheric gases, the radiative transfer and the dynamic modeling of transport phenomena, and photochemistry in the atmosphere and more specialized treatments of remote sensing and in situ techniques used to gather data on the atmosphere from the ground as well as from airborne and space platforms in passive and active modes.

Most of the papers are clearly presented and are well documented with general as well as specific references on the various topics. There are, however, some inhomogeneities among the presentations and redundancies in the presented material. The book will be useful not necessarily only to fluent French read- 